\title{
Unsteady flow over an expanding cylinder in a nanofluid containing gyrotactic microorganisms
}

\begin{tabular}{|r|l|}
\hline Journal: & Canadian Journal of Physics \\
\hline Manuscript ID & cjp-2015-0335.R2 \\
\hline Manuscript Type: & Article \\
\hline Date Submitted by the Author: & 07-Jan-2016 \\
\hline Complete List of Authors: & $\begin{array}{l}\text { Chen, Hui; Fuzhou University, School of Mechanical Engineering and } \\
\text { Automation } \\
\text { Liang, Hongxing; Fuzhou University, School of Mechanical Engineering and } \\
\text { Automation } \\
\text { Xiao, Tianli; Fuzhou University, School of Mechanical Engineering and } \\
\text { Automation } \\
\text { Du, Heng; Fuzhou University, School of Mechanical Engineering and } \\
\text { Automation } \\
\text { Shen, Ming; Fuzhou University, College of Mathematics and Computer } \\
\text { Science }\end{array}$ \\
\hline Keyword: & $\begin{array}{l}\text { nanofluids, expanding cylinder, gyrotactic microorganisms, } \\
\text { stretching/shrinking, unsteady flow }\end{array}$ \\
\hline
\end{tabular}




\title{
Unsteady flow over an expanding cylinder in a nanofluid containing gyrotactic microorganisms
}

\author{
Hui Chen ${ }^{1 ⿴ 囗 十)}$ \\ Hongxing Liang ${ }^{1}$ \\ Tianli $\mathrm{Xiao}^{1}$ \\ Heng $\mathrm{Du}^{1}$ \\ Ming Shen ${ }^{2}$ \\ ${ }^{1}$ School of Mechanical Engineering and Automation, Fuzhou University, 350108 \\ Fuzhou, Fujian, China \\ ${ }^{2}$ College of Mathematics and Computer Science, Fuzhou University, 350108 \\ Fuzhou, Fujian, China \\ PACS Nos: 44.20.+b, 47.15.Cb.
}




\begin{abstract}
In this paper, an analysis is made for the unsteady flow due to an expanding cylinder in a nanofluid which contains both nanoparticles and gyrotactic microoganisms with suction. The nonlinear system of partial differential equations is transformed to high order non-linear and ordinary differential equations by similarity transformations and then solved numerically using a shooting method with fourth-fifth-order Runge-Kutta integration technique. Besides, the influences of significant physical parameters on the distributions of the velocity, temperature, nanoparticle volume fraction, as well as the density of motile microorganisms are graphically presented and discussed in detail. It is found that dual solutions exist for both stretching and shrinking cases and the range of dual solutions increases with the strength of the expansion. The results also indicate that larger bioconvection Peclet number and smaller Schmidt number lead to higher the concentrate of microorganisms and thicker boundary layer thickness.
\end{abstract}

Keywords: Nanofluid, Gyrotactic microorganisms, Expanding cylinder, Stretching/Shrinking

PACS Nos: 44.20.+b, 47.15.Cb.

\title{
1 Introduction
}

Poor thermal conductivity of conventional fluids such as water, ethylene glycol, and engine oil, limit the performance enhancement and the compactness of many thermal applications. An innovative technique for improving heat transfer by using nanoparticles (diameter less than $50 \mathrm{~nm}$ ) in a based fluid has been used extensively recently. It was first introduced by Choi [1] who proposed the term 'nanofluid' to describe the liquid suspension, which has the unique physical and chemical properties due to the ultrafine of nanoparticles [2]. After the pioneering work, nanofluids have attracted enormous interest from researchers because of its many significant applications such as medical applications, chemical engineering, transportations, electronics as well as manufacturing and food. A comprehensive literature on the topic of nanofluid can be found in the famous book by Das et al. [3] and Nield and Bejan [4], and in the review papers by Buongiorno [5], Kakaç and Pramuanjaroenkij [6], Wong and De Leon [7], Fan and Wang [8], Mahian et al. [9], Hussein et al. [10], and Mahdi et al. [11].

It should be mentioned that the nanoparticles in the base fluid are unstable, which may result in aggregating and decreasing of thermal conductivity of nanofluids. Introducing the upswimming microorganisms is a novel method presented by Kuznetsov [12] to solve unstable problem and avoid nanoparticles from agglomerating and aggregating. Thus, The nanofluid containing both nanoparticles and gyrotactic microorganisms has drawn considerable attention of several researchers such as Tham et al. [13], Zaimi et al. [14], Khan et al. [15, 16], Mutuku et al. [17], Xu and Pop [18] and many others. In a nanofluid containing nanoparticle and motile microorganisms, it should be also mentioned that the nanoparticles are not self-propelled and their motion is driven by Brownian diffusion and thermophoresis occurring in the nanofluids which explored by Buongiorno [5].

During the past few years, the problem of nanofluid over a cylinder have been explored by a great number of researchers due to its many relevant applications such as heat exchangers, nuclear reactor fuel rods and steel suspension bridge cables. It seems that Nazar et al. [19] have first studied the mixed convection flow past a circular cylinder embedded in a porous medium saturated by a nanofluid. They reported that heating the cylinder delays boundary layer separation and cooling the cylinder brings the boundary layer separation point nearer to the lower stagnation point. Later Grosan and Pop [20] numerically examined the steady axisymmetric mixed convection flow and heat transfer characteristics past a vertical cylinder immersed in a nanofluid. It was found that dual solutions exist when the cylinder surface is cooled. Ever since, much more attention has been drawn to this type of flow such as Tham et al. [21, 22], Rashad et al. [23, 25], Prasad et al. [25], El-Kabeir et al. [26], Rohni et al. [27], and Ashorynejad et al. [28].

All the above investigations deal with only the steady flow of the nanofluids along the cylinder. In reality the flow and heat transfer problems may be unsteady, due to motion of cylinder surface or the sudden changes of surface temperature. The steady (fully developed) flow is results of the development of viscous unsteady

Hui Chen, Hongxing Liang, Tianli Xiao and Heng Du. School of Mechanical Engineering and Automation, Fuzhou University, Fuzhou, China.

Ming Shen. College of Mathematics and Computer Science, Fuzhou University, Fuzhou, China.

Corresponding author: Hui Chen (e-mail: felixceng@fzu.edu.cn) 
flow after a certain instant of time in most cases. However, very little work on unsteady nanofluids flow along the cylinder has been reported in the literatures including Zaimi et al. [29], Elbashbeshy et al. [30], Chamkha et al. [31] , and Sarkar et al. [32]. It may be remarked that the innovative method on adding of gyrotactic microorganisms to avoid nanoparticles from agglomerating and aggregating, has not been considered in the problem of unsteady nanofluid flow over cylinder.

The major of the present paper is to investigate the unsteady flow due to an expanding cylinder in a nanofluid which containing both nanoparticles and gyrotactic microoganisms. It should be mentioned that the problems of unsteady flow in cylindrical coordinates are important issues in many biomicrosystems, although several aspects of nanofluid containing gyrotactic microorganisms have been presented. The present study is to extend the work investigated by Zaimi et al. [29] to the case of the unsteady flow due to an expanding cylinder in a nanofluid using Buongiorno's model. By means of similarity reductions, a set of four coupled nonlinear equations are solved numerically. Besides, the effects of the governing parameters on the velocity, temperature, nanoparticle volume fraction, as well as the density of motile microorganisms profiles are graphically presented and discussed. As far as we know, there seems to be no attempts in literature to consider this problem and we believe that the paper is original and the major results are new.

\section{Flow analysis}

We consider the unsteady laminar flow of a viscous and incompressible nanofluid containing both nanoparticles and gyrotactic microorganisms over an expanding stretching/shrinking cylinder. The physical model and coordinate system are shown in Fig. 1. The diameter of the cylinder is assumed as a function of time. It is assumed that the unsteady and incompressible nanofluid is without body force. Under these assumptions, the governing equations for the continuity, momentum, energy, nanoparticle volume fraction and microorganisms in the vectorial form $[5,12]$ are given in the following form:

$$
\begin{aligned}
& \nabla \cdot \mathbf{V}=0 \\
& \frac{\partial \mathbf{V}}{\partial t}+(\mathbf{V} \cdot \nabla) \mathbf{V}=-\frac{1}{\rho} \nabla p+\nu \nabla^{2} \mathbf{V} \\
& \frac{\partial T}{\partial t}+\mathbf{V} \cdot \nabla T=\alpha \nabla^{2} T+\tau\left[D_{B} \nabla T \cdot \nabla C+\left(D_{T} / T_{\infty}\right) \nabla T \cdot \nabla T\right] \\
& \frac{\partial C}{\partial t}+\mathbf{V} \cdot \nabla C=D_{B} \nabla^{2} C+\left(D_{T} / T_{\infty}\right) \nabla^{2} T \\
& \frac{\partial N}{\partial t}+\nabla \cdot j=0
\end{aligned}
$$

where $\mathbf{V}, T, C, N$ denote the velocity vector of nanofluid flow, temperature, nanoparticle concentration and the concentration of microorganisms, respectively. $p$ is the pressure, $\nu$ is the kinematic viscosity, $k$ is the porosity

Fig. 1. Physical model and coordinate system

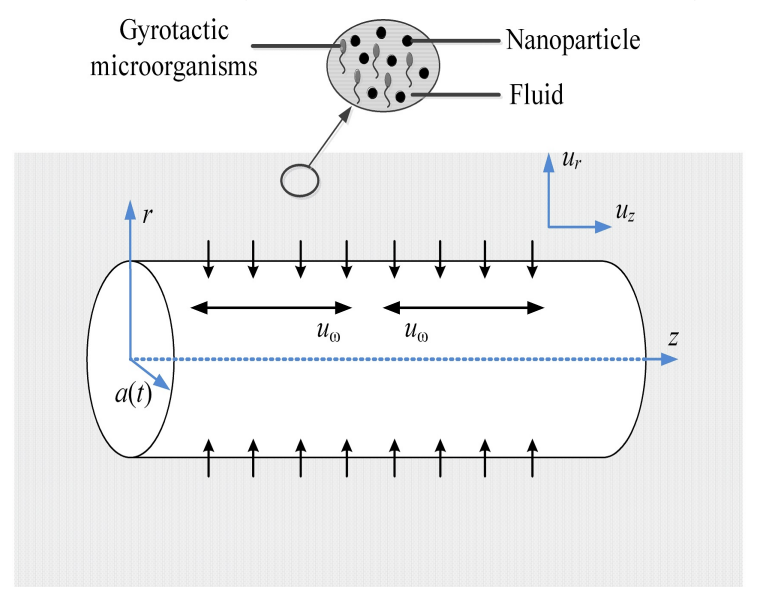


of the medium, $\rho$ is the fluid density, $\alpha$ is the thermal diffusivity of the nanofluid, $\tau=(\rho c)_{p} /(\rho c)_{f}$ is ratio between the heat capacity of the nanoparticle and the heat capacity of fluid, $D_{B}$ is the Brownian diffusion coefficient, $D_{T}$ is the thermophoretic diffusion coefficient and $j$ is the flux of microorganisms which is defined as

$$
j=N \mathbf{V}+N \tilde{\mathbf{V}}-D_{n} \nabla N,
$$

in which $\tilde{\mathbf{V}}=\left(b W_{c} / \Delta C\right) \nabla C$ is the velocity vector relating to the cell swimming in nanofluids with $D_{n}$ is the diffusivity of microorganisms, $b$ is the chemotaxis constant, $\Delta C=C_{w}-C_{\infty}$ and $W_{c}$ is the maximum cell swimming speed.

In cylindrical polar coordinates, the axis of the cylinder is taken along the $z$-axis, while the $r$-axis is taken in the radial direction. Based on the axisymmetric flow assumptions, the equations (1)-(5) can be written as

$$
\begin{aligned}
& \frac{\partial}{\partial r}\left(r v_{r}\right)+\frac{\partial}{\partial z}\left(r v_{z}\right)=0, \\
& \frac{\partial v_{r}}{\partial t}+v_{r} \frac{\partial v_{r}}{\partial r}+v_{z} \frac{\partial v_{r}}{\partial z}=-\frac{1}{\rho} \frac{\partial p}{\partial r}+\nu\left(\frac{\partial^{2} v_{r}}{\partial r^{2}}+\frac{1}{r} \frac{\partial v_{r}}{\partial r}+\frac{\partial^{2} v_{r}}{\partial z^{2}}-\frac{v_{r}}{r^{2}}\right) \\
& \frac{\partial v_{z}}{\partial t}+v_{r} \frac{\partial v_{z}}{\partial r}+v_{z} \frac{\partial v_{z}}{\partial z}=-\frac{1}{\rho} \frac{\partial p}{\partial z}+\nu\left(\frac{\partial^{2} v_{z}}{\partial r^{2}}+\frac{1}{r} \frac{\partial v_{z}}{\partial r}+\frac{\partial^{2} v_{z}}{\partial z^{2}}\right) \\
& \frac{\partial T}{\partial t}+v_{r} \frac{\partial T}{\partial r}+v_{z} \frac{\partial T}{\partial z}=\alpha\left(\frac{\partial^{2} T}{\partial r^{2}}+\frac{1}{r} \frac{\partial T}{\partial r}+\frac{\partial T^{2}}{\partial z^{2}}\right)+\tau\left(D_{B} \frac{\partial C}{\partial r} \frac{\partial T}{\partial r}+\frac{\partial C}{\partial z} \frac{\partial T}{\partial z}\right) \\
&+\frac{\tau D_{T}}{T_{\infty}}\left[\left(\frac{\partial T}{\partial r}\right)^{2}+\left(\frac{\partial T}{\partial z}\right)^{2}\right], \\
& \frac{\partial C}{\partial t}+v_{r} \frac{\partial C}{\partial r}+v_{z} \frac{\partial C}{\partial z}=D_{B}\left(\frac{\partial^{2} C}{\partial r^{2}}+\frac{1}{r} \frac{\partial C}{\partial r}+\frac{\partial C^{2}}{\partial z^{2}}\right)+\frac{D_{T}}{T_{\infty}}\left(\frac{\partial^{2} T}{\partial r^{2}}+\frac{1}{r} \frac{\partial T}{\partial r}+\frac{\partial T^{2}}{\partial z^{2}}\right), \\
& \frac{\partial N}{\partial t}+v_{r} \frac{\partial N}{\partial r}+v_{z} \frac{\partial N}{\partial z}=-\frac{b W_{c}}{\Delta C}\left[N\left(\frac{\partial^{2} C}{\partial r^{2}}+\frac{1}{r} \frac{\partial C}{\partial r}+\frac{\partial C^{2}}{\partial z^{2}}\right)+\frac{\partial C}{\partial r} \frac{\partial N}{\partial r}+\frac{\partial C}{\partial z} \frac{\partial N}{\partial z}\right] \\
&+D_{n}\left(\frac{\partial^{2} N}{\partial r^{2}}+\frac{1}{r} \frac{\partial N}{\partial r}+\frac{\partial N^{2}}{\partial z^{2}}\right) .
\end{aligned}
$$

The boundary conditions for the physical problem are given by

$$
\begin{aligned}
& v_{r}=-\frac{2 \nu}{a_{0} \sqrt{1-\beta t}}, \quad v_{z}=\frac{4 \nu z \varepsilon}{a_{0}^{2}(1-\beta t)}, \\
& T=T_{w}, \quad D_{B} \frac{\partial C}{\partial r}+\frac{D_{T}}{T_{\infty}} \frac{\partial T}{\partial r}=0, N=N_{w} \text { at } r=a(t), \\
& v_{z} \rightarrow 0, \quad T \rightarrow T_{\infty}, \quad C \rightarrow C_{\infty} \quad N \rightarrow 0 \text { as } r \rightarrow \infty .
\end{aligned}
$$

Here $a(t)=a_{0} \sqrt{1-\beta t}$ is the varying cylinder diameter, where $\beta$ is a constant with $\beta>0$ for a contracting cylinder and $\beta<0$ for a expanding cylinder. To convent the governing equations into ordinary differential equations, using the similarity variables [33]

$$
\begin{aligned}
& \eta=\frac{r^{2}}{a_{0}^{2}(1-\beta t)}, \quad v_{r}=-\frac{2 \nu f(\eta)}{r}, \quad v_{z}=\frac{4 \nu z \eta f^{\prime}(\eta)}{r^{2}} \\
& \theta(\eta)=\frac{T-T_{\infty}}{T_{w}-T_{\infty}}, \quad \phi(\eta)=\frac{C-C_{\infty}}{C_{w}-C_{\infty}}, \quad \omega(\eta)=\frac{N}{N_{w}} .
\end{aligned}
$$

Since the equation (7) is automatically satisfied and the longitudinal pressure gradient is zero, Eqs. (8)-(12) are transformed into

$$
\begin{aligned}
& \eta f^{\prime \prime \prime}+f^{\prime \prime}+f f^{\prime \prime}-f^{\prime 2}-\lambda\left(f^{\prime}+\eta f^{\prime \prime}\right)=0, \\
& \eta \theta^{\prime \prime}+\theta^{\prime}+P_{r}\left(f \theta^{\prime}-\lambda \eta \theta^{\prime}\right)+\eta\left(N_{b} \phi^{\prime} \theta^{\prime}+N_{t} \theta^{2}\right)=0, \\
& \eta \phi^{\prime \prime}+\phi^{\prime}+L_{e}\left(f \phi^{\prime}-\lambda \eta \phi^{\prime}\right)+\frac{N_{t}\left(\eta \theta^{\prime \prime}+\theta^{\prime}\right)}{N_{b}}=0, \\
& \eta \omega^{\prime \prime}+\omega^{\prime}-P_{e}\left(\eta \omega \phi^{\prime \prime}+\omega \phi^{\prime}+\eta \omega^{\prime} \phi^{\prime}\right)+S_{c}\left(f \omega^{\prime}-\lambda \eta \omega^{\prime}\right)=0,
\end{aligned}
$$


and the boundary conditions become

$$
\begin{aligned}
& f(1)=1, \quad f^{\prime}(1)=\varepsilon, \quad \theta(1)=1, \quad \omega(1)=1, \quad N_{b} \phi^{\prime}(1)+N_{t} \theta^{\prime}(1)=0, \\
& f^{\prime}(\infty)=\theta(\infty)=\phi(\infty)=\omega(\infty)=0
\end{aligned}
$$

where prime denotes differentiation with respect to $\eta$. In the above equations, $\lambda=a_{0}^{2} \beta / 4 \nu$ is the unsteadiness parameter for a contracting cylinder which displays the strength of the contraction. $\varepsilon$ is the stretching/shrinking parameter and $P_{r}$ is the Prandtl number. $N_{t}$ and $N_{b}$ are the thermophoresis and Brownian motion parameters respectively which depend on thermal diffusivity $[34,35], L_{e}$ is the Lewis number, $P_{e}$ is the bioconvection Peclet number and $S_{c}$ is the Schmidt number, which are given by

$$
\begin{aligned}
& P_{r}=\frac{\nu}{\alpha}, \quad N_{t}=\frac{\tau D_{T}\left(T_{w}-T_{\infty}\right)}{\alpha T_{\infty}}, \\
& N_{b}=\frac{\tau D_{B}\left(C_{w}-C_{\infty}\right)}{\alpha}, \quad L_{e}=\frac{\nu}{D_{B}}, \quad P_{e}=\frac{b w_{c}}{D_{n}}, \quad S_{c}=\frac{\nu}{D_{n}} .
\end{aligned}
$$

The quantities of physical interest are the skin friction coefficient $C_{f}$, the local Nusselt number $N u_{x}$, the local Sherwood number $S h_{x}$ and the local density of the motile microorganisms $N n_{x}$, which are defined as [29]

$$
\begin{aligned}
C_{f} & =\frac{\mu\left(\partial v_{z} / \partial r\right)_{r=a(t)}}{\rho v_{z w}^{2} / 2}, \quad N u_{x}=\frac{-a_{0} k(1-\beta t)^{1 / 2}(\partial T / \partial r)_{r=a(t)}}{2 k\left(T_{w}-T_{\infty}\right)} \\
S h_{x} & =\frac{-a_{0} D_{B}(1-\beta t)^{1 / 2}(\partial C / \partial r)_{r=a(t)}}{2 D_{B}\left(C_{w}-C_{\infty}\right)}, \quad N n_{x}=\frac{-a_{0} D_{n}(1-\beta t)^{1 / 2}(\partial N / \partial r)_{r=a(t)}}{2 D_{n} N_{w}} .
\end{aligned}
$$

Using the applying the similarity transformation (16) and (17), we obtain

$$
\begin{aligned}
& C_{f} \frac{z \varepsilon^{2}}{a(t)}=f^{\prime \prime}(1), \quad N u_{x}=-\theta^{\prime}(1), \\
& S h_{x}=-\phi^{\prime}(1), \quad N n_{x}=-\omega^{\prime}(1) .
\end{aligned}
$$

\section{Result and discussions}

Numerical solutions to the nonlinear ordinary differential equations (18)-(21) with the boundary conditions (22)-(23) are obtained by using a shooting method with fourth-fifth-order Runge-Kutta integration technique. The appropriate finite value of boundary layer thickness $\eta_{\infty}\left(\eta_{\infty}=15\right)$ is chosen to acquire sufficiently accurate (error less than $10^{-5}$ ) results. Then a Newton-Raphson iteration is applied to assure the iteration convergence required to satisfy the boundary conditions at infinity. Dual solutions are obtained by setting different initial guesses of $f^{\prime \prime}(1),-\theta^{\prime}(1),-\phi^{\prime}(1)$ and $-\omega^{\prime}(1)$ where all the velocity, temperature, nanoparticle concentration and the concentration of microorganisms profiles satisfy the infinity boundary conditions (23) asymptotically but with different shapes. For the accuracy verification of the applied numerical method, comparisons of the skin friction coefficient $f^{\prime \prime}(1)$ for $\varepsilon=-1$ are made with the available results of Wan Zaimi et al. [36]. The results are presented in Table 1 and the excellent agreement is noted. Moreover, some typical results of $f^{\prime \prime}(1),-\theta^{\prime}(1)$ and $-\omega^{\prime}(1)$ of the present study are shown in Table 2 . It is note that the values of $-\phi^{\prime}(1)$ can be obtained from the boundary condition (22).

Table 1. Comparison with previously published data for the values of $f^{\prime \prime}(1)$ when $\varepsilon=-1$ (shrinking case)

\begin{tabular}{crrrrr}
\hline \multirow{2}{*}{$\lambda$} & \multicolumn{2}{c}{ Wan Zaimi et al. } & & \multicolumn{2}{c}{ Present work } \\
\cline { 2 - 3 } \cline { 5 - 6 } & First sol & Second sol & & First sol & Second sol \\
\hline-4 & 4.7879 & -34.6866 & & 4.78798 & -34.68664 \\
-3.5 & 4.2610 & -25.3598 & & 4.26102 & -25.35983 \\
-3 & 3.7255 & -17.5991 & & 3.72552 & -17.59912 \\
-2.5 & 3.1761 & -11.3068 & & 3.17615 & -11.30678 \\
-2 & 2.6012 & -6.3803 & & 2.60122 & -6.38004 \\
\hline
\end{tabular}


Talbe 2. Numerical values of $f^{\prime \prime}(1),-\theta^{\prime}(1)$ and $-\omega^{\prime}(1)$ for various values of $\varepsilon$ and $\lambda$ when $\operatorname{Pr}=6.2, N_{t}=3.1, N_{b}=3.1, L_{e}=2, P_{e}=1, S_{c}=1$

\begin{tabular}{|c|c|c|c|c|c|c|c|}
\hline \multirow[t]{2}{*}{$\varepsilon$} & \multirow[t]{2}{*}{$\lambda$} & \multicolumn{2}{|c|}{$f^{\prime \prime}(1)$} & \multicolumn{2}{|c|}{$-\theta^{\prime}(1)$} & \multicolumn{2}{|c|}{$-\omega^{\prime}(1)$} \\
\hline & & First sol & Second sol & First sol & Second sol & First sol & Second sol \\
\hline & -3 & 3.72552 & -17.59912 & 17.31518 & 17.04931 & -12.52810 & $\begin{array}{l}-12.89707 \\
\end{array}$ \\
\hline \multirow[t]{3}{*}{-1} & -2 & 2.60122 & -6.38004 & 12.97483 & 12.77171 & -9.33659 & -9.58686 \\
\hline & -1 & 1.00006 & 0.08412 & 8.431026 & 8.377436 & -6.14246 & -6.19341 \\
\hline & -3 & 1.93373 & -20.05711 & 17.42158 & 17.15534 & -12.55719 & -12.93786 \\
\hline \multirow[t]{3}{*}{-0.5} & -2 & 1.40729 & -8.30500 & 13.12004 & 12.91153 & -9.37511 & -9.64797 \\
\hline & -1 & 0.83514 & -1.63337 & 8.69476 & 8.56770 & -6.18298 & -6.32863 \\
\hline & -3 & 6.95988 & -22.54364 & 17.52511 & 17.25976 & -12.58648 & -12.97636 \\
\hline \multirow[t]{3}{*}{0} & -2 & 2.15681 & -10.23227 & 13.25785 & 13.04863 & -9.41433 & -9.70278 \\
\hline & -1 & 1.93952 & -2.98030 & 8.90958 & 8.77248 & 6.24103 & -6.42112 \\
\hline & -3 & -2.06235 & -25.07129 & 17.62606 & 17.36248 & -12.61585 & -13.01300 \\
\hline \multirow[t]{3}{*}{0.5} & -2 & -1.58293 & -12.19980 & 13.38965 & 13.18228 & -9.45371 & -9.75295 \\
\hline & -1 & -1.12342 & -4.35223 & 9.10275 & 8.96811 & -6.30034 & -6.49620 \\
\hline & -3 & -4.24278 & -27.64898 & 17.72467 & 17.46346 & -12.64523 & -13.04806 \\
\hline \multirow[t]{2}{*}{1} & -2 & -3.31709 & -14.22902 & 13.51640 & 13.31225 & -9.49299 & -9.79964 \\
\hline & -1 & -2.45157 & -5.82261 & 9.28127 & 9.15232 & -6.35882 & -6.56178 \\
\hline
\end{tabular}

Figs. 2-4 display the variation of skin friction coefficients $f^{\prime \prime}(1)$, local Nusselt number $-\theta^{\prime}(1)$ and local density of motile microorganisms $-\omega^{\prime}(1)$ with $\varepsilon$ for different values of $\lambda$. It is observed that there are dual solutions (first solution and second solution) when $\varepsilon>\varepsilon_{c}$, and one solution when $\varepsilon=\varepsilon_{c}$, but no solution is found in the range of $\varepsilon<\varepsilon_{c}$. The range of the dual solution increases as the strength of

Fig. 2. Variation of the skin friction coefficient $f^{\prime \prime}(1)$ with $\varepsilon$ for different values of $\lambda$ when $\operatorname{Pr}=$ 6.2, $N_{t}=3.1, N_{b}=3.1, L_{e}=2, P_{e}=1, S_{c}=1$

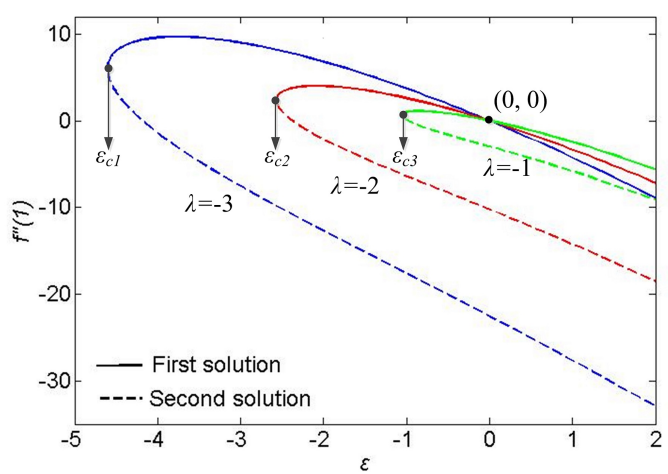

Fig. 3. Variation of the local Nusselt number $-\theta^{\prime}(1)$ with $\varepsilon$ for different values of $\lambda$ when $\operatorname{Pr}=$ 6.2, $N_{t}=3.1, N_{b}=3.1, L_{e}=2, P_{e}=1, S_{c}=1$

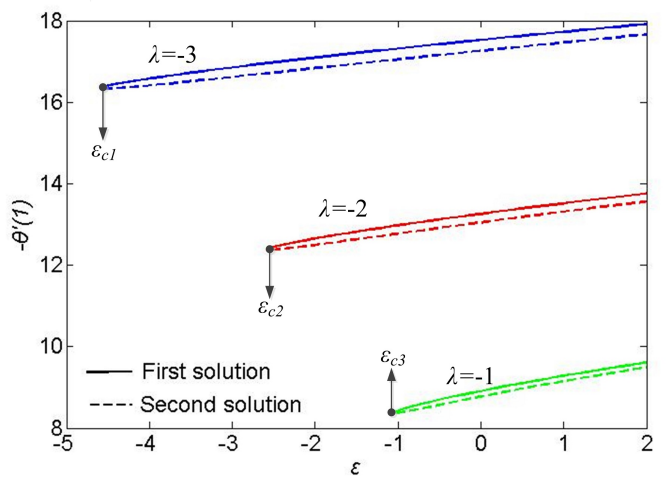

Fig. 4. Variation of the local density of the motile microorganisms $-\omega^{\prime}(1)$ with $\varepsilon$ for different values of $\lambda$ when $\operatorname{Pr}=6.2, N_{t}=3.1, N_{b}=3.1, L_{e}=2$, $P_{e}=1, S_{c}=1$

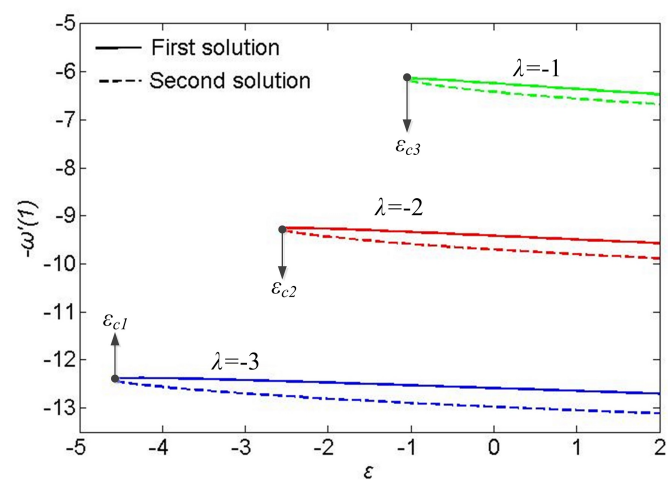


expansion $|\lambda|$ increases. It is also seen from Figs. 2 and 4 that $f^{\prime \prime}(1)$ and $-\omega^{\prime}(1)$ decrease with an increase in $\varepsilon$ for the two solutions. In contrast, $-\theta^{\prime}(1)$ increases with $\varepsilon$ which indicates that the stretching enhances the heat transfer at the surface, but the shrinking inhibits the effect of heat transfer at the surface. It is interesting to noted from Fig. 2 that the value of $f^{\prime \prime}(1)$ always equals to zero when $\varepsilon=0$ which is consistent with the result reported by Bachok et al. [37]. As shown in Fig. 3, the local Nusselt number is consistently higher with higher value of $|\lambda|$. It is worth noting that the local Nusselt number is always positive, i.e. the heat is transferred from the hot surface to the cold fluid, which is consistent with physical fact. It is shown that local density of the motile microorganisms decreases dramatically with $|\lambda|$ in Fig. 4, while the effect of strength of expansion $|\lambda|$ on local Sherwood number is not remarkable.

The influences of $\lambda$ on velocity, temperature, nanoparticle concentration and the concentration of microorganisms profiles are presented in Figs. 5-9. It is clear that the far field boundary conditions (23) are satisfied asymptotically, which shows the validity of the results. All of the boundary layer thicknesses of first solution are smaller than that of second solution for a particular value of the unsteady parameter $\lambda$. As shown in Fig. 5 , the velocity gradient at the surface is positive for the first solution and negative for the second solution for the shrinking case $(\varepsilon=-0.5)$, which is accordant with the results presented in Fig. 2. We expect the first solution to be the physically realizable solution. For the first solution, the velocity profile increases as $|\lambda|$ increases, and the velocity boundary layer thickness decreases with $|\lambda|$. Moreover, the velocity gradient at the surface increases with increasing $|\lambda|$, and in consequent increase the skin friction coefficient. For the stretching case $(\varepsilon=2)$ as shown in Fig. 6, the velocity profile and boundary layer thickness of first solution decrease with increasing $|\lambda|$, i.e. the velocity gradient at the surface is decreased. This phenomenon indicates that the effect of strength of expansion on the first solution of velocity profile for shrinking and stretching case is opposite. These results are also agreed with the result showed in Fig. 2. It is observed from the profiles presented in Figs. 7-9 that the

Fig. 5. Velocity profiles $f^{\prime}(\eta)$ for different values of $\lambda$ when $\operatorname{Pr}=6.2, N_{t}=3.1, N_{b}=3.1, L_{e}=2$, $P_{e}=1, S_{c}=1, \varepsilon=-0.5$ (shrinking case)

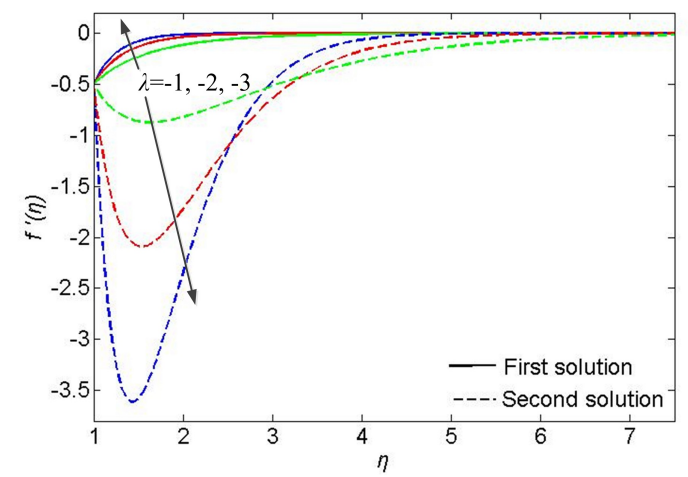

Fig. 7. Temperature profiles $\theta(\eta)$ for different values of $\lambda$ when $\operatorname{Pr}=6.2, N_{t}=3.1, N_{b}=3.1$, $L_{e}=2, P_{e}=1, S_{c}=1, \varepsilon=-0.5$

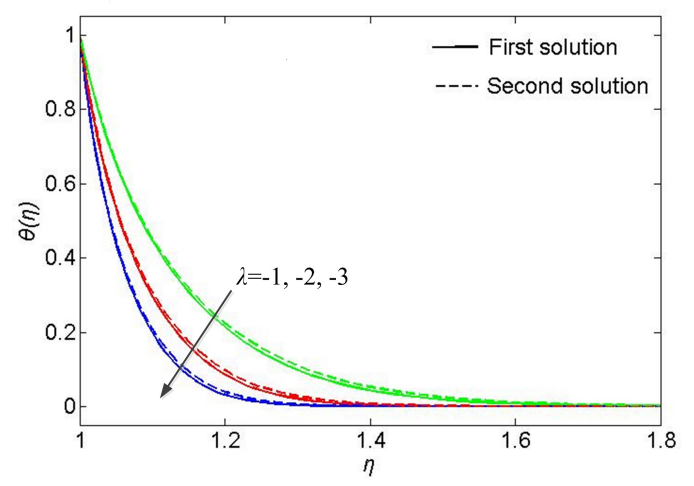

Fig. 6. Velocity profiles $f^{\prime}(\eta)$ for different values of $\lambda$ when $\operatorname{Pr}=6.2, N_{t}=3.1, N_{b}=3.1, L_{e}=2$, $P_{e}=1, S_{c}=1, \varepsilon=2$ (stretching case)

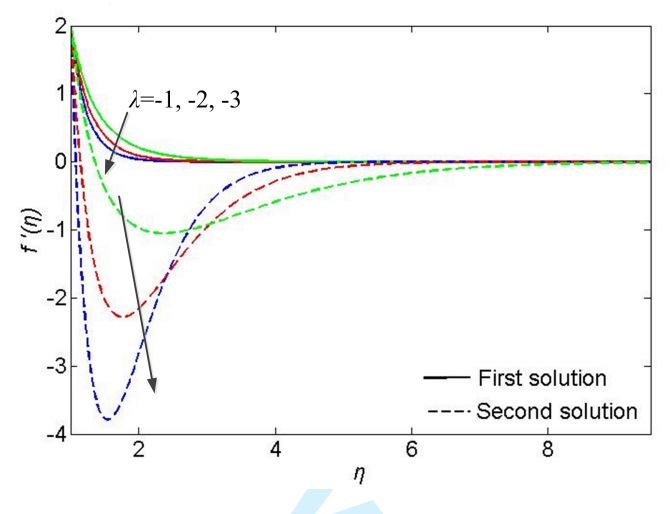

Fig. 8. Nanoparticle valume fraction profiles $\phi(\eta)$ for different values of $\lambda$ when $\operatorname{Pr}=6.2, N_{t}=3.1$, $N_{b}=3.1, L_{e}=2, P_{e}=1, S_{c}=1, \varepsilon=-0.5$

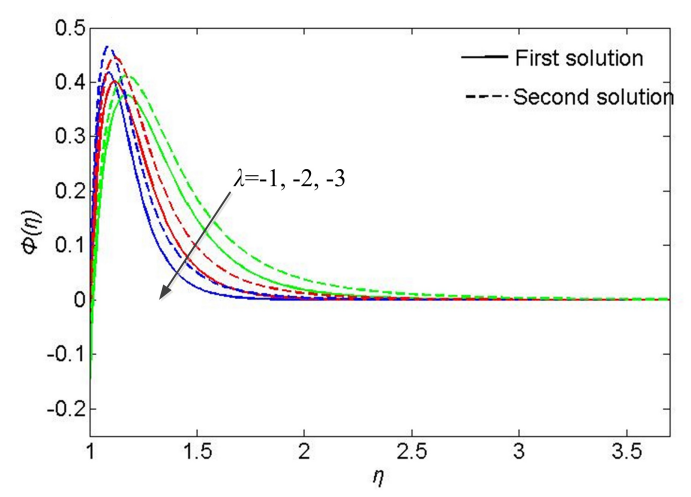


Fig. 9. Density of motile microorganisms profiles $\omega(\eta)$ for different values of $\lambda$ when $\operatorname{Pr}=6.2, N_{t}=$ $3.1, N_{b}=3.1, L_{e}=2, P_{e}=1, S_{c}=1, \varepsilon=-0.5$

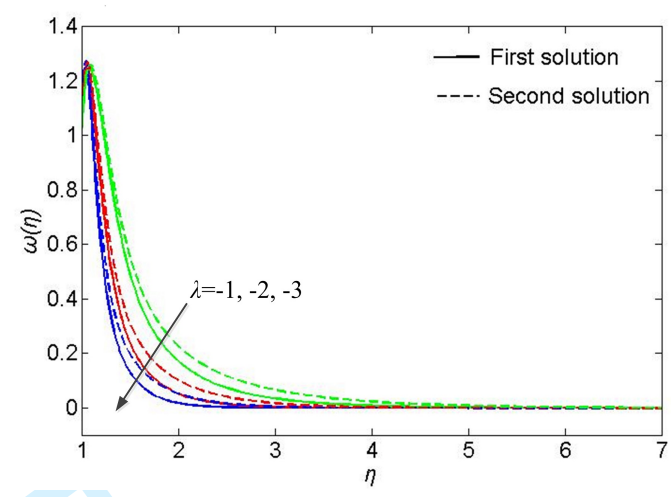

boundary layer thicknesses of temperature, nanoparticle concentration and concentration of microorganisms for the shrinking case decrease with an increase in $|\lambda|$ for the two solutions. Different from the temperature which is monotone decreasing with $\eta$, the nanoparticle concentration and concentration of microorganisms increase rapidly to a maximum value when $\eta$ is small and then decrease to the far field conditions. It is noteworthy that the difference between the first and second solutions is small, which is also consistent with the results presented in Figs. 3 and 4. In addition, for the stretching case, temperature, nanoparticle concentration and concentration of microorganisms profiles are toward the same trend with the shrinking case, so that we do not present the corresponding graphs in this section.

Figs. 10-15 show the effect of the Brownian motion parameter $N_{b}$ and the thermophoresis parameter $N_{t}$ on the temperature, nanoparticle concentration and concentration of microorganisms profiles for the shrinking case. As shown in these figures, the first solutions approach to the far field conditions are faster than the second solutions, i.e. the boundary layer thicknesses of the first solutions are smaller than that of the second solutions. It is noted from Fig. 10 that the temperature and the thermal boundary layer thickness increase as $N_{b}$ increases. In contrast, the nanoparticle concentration and concentration of microorganisms decrease with increasing $N_{b}$ as illustrated in Figs. 11 and 12. The thermophoresis parameter $N_{t}$ exhibits an opposite effect on these profiles compared with Brownian motion parameter $N_{b}$ as shown in Figs. 13-15, which is in agreement with the physical fact.

Fig. 10. Temperature profiles $\theta(\eta)$ for different values of $N_{b}$ when $\lambda=-2 \operatorname{Pr}=6.2, N_{t}=3.1$, $L_{e}=2, P_{e}=1, S_{c}=1, \varepsilon=-0.5$

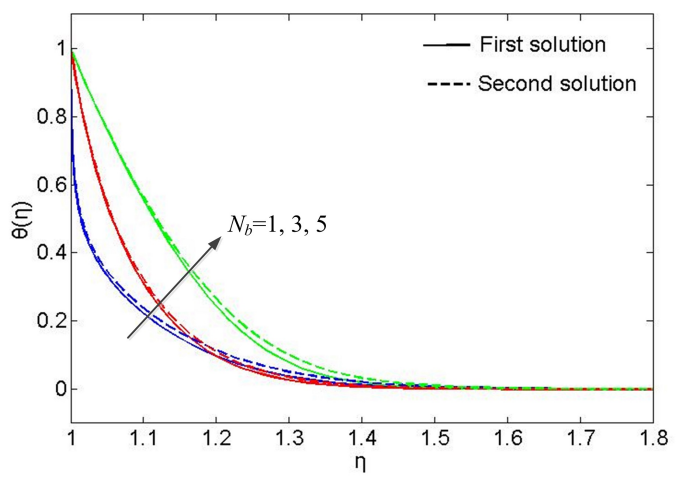

Fig. 11. Nanoparticle valume fraction profiles $\phi(\eta)$ for different values of $N_{b}$ when $\lambda=-2$ $\operatorname{Pr}=6.2, N_{t}=3.1, L_{e}=2, P_{e}=1, S_{c}=1$, $\varepsilon=-0.5$

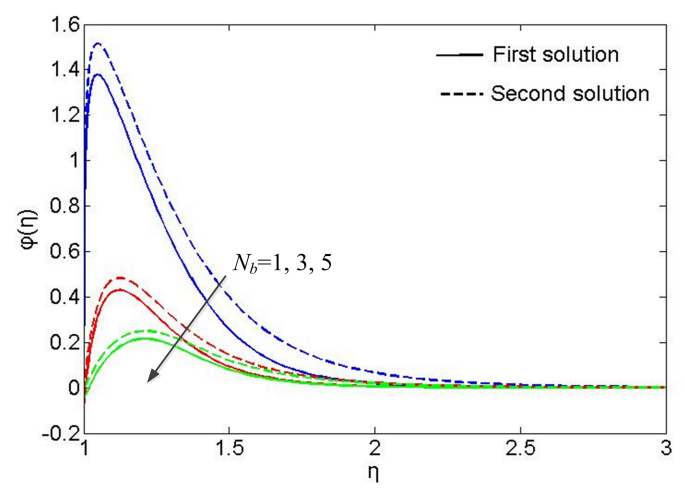


Fig. 12. Density of motile microorganisms profiles $\omega(\eta)$ for different values of $N_{b}$ when $\lambda=-2 \operatorname{Pr}=$ $6.2, N_{t}=3.1, L_{e}=2, P_{e}=1, S_{c}=1, \varepsilon=-0.5$

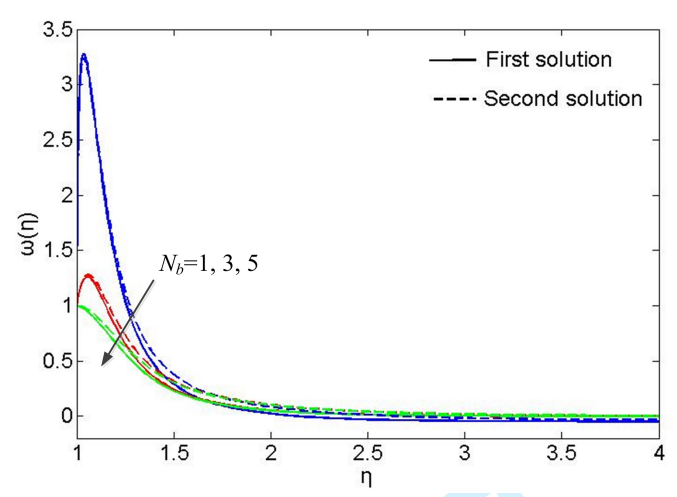

Fig. 14. Nanoparticle valume fraction profiles $\phi(\eta)$ for different values of $N_{t}$ when $\lambda=-2$ $\operatorname{Pr}=6.2, N_{b}=3.1, L_{e}=2, P_{e}=1, S_{c}=1$, $\varepsilon=-0.5$

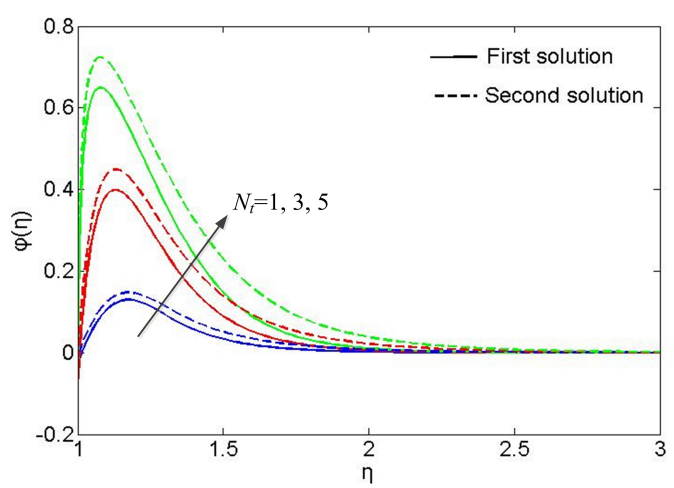

Fig. 13. Temperature profiles $\theta(\eta)$ for different values of $N_{t}$ when $\lambda=-2 \operatorname{Pr}=6.2, N_{b}=3.1$, $L_{e}=2, P_{e}=1, S_{c}=1, \varepsilon=-0.5$

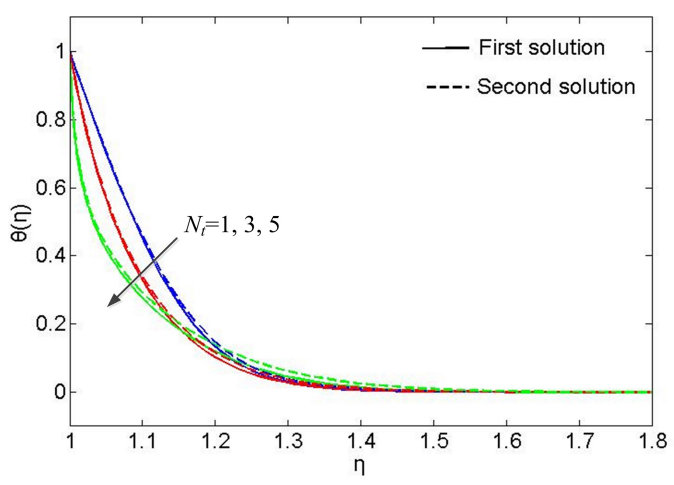

Fig. 15. Density of motile microorganisms profiles $\omega(\eta)$ for different values of $N_{t}$ when $\lambda=-2 \operatorname{Pr}=$ $6.2, N_{b}=3.1, L_{e}=2, P_{e}=1, S_{c}=1, \varepsilon=-0.5$

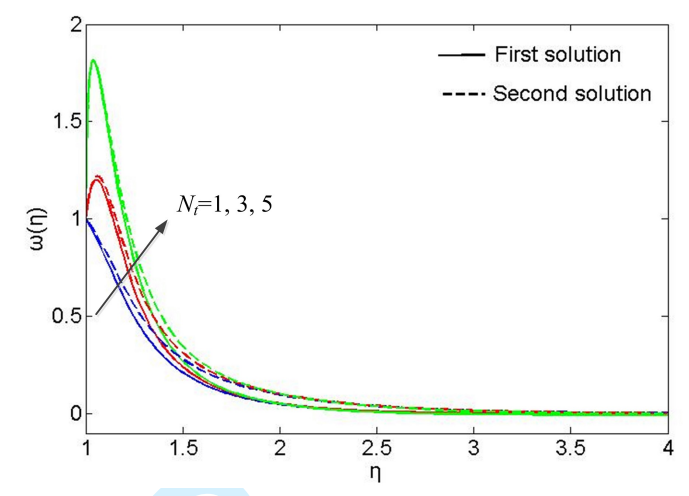

It is noticed that bioconvection Peclet number $P_{e}$ and Schmidt number $S_{c}$ have only effects on the concentration of microorganisms profile $\omega(\eta)$ from the governing equations. As shown in Fig. 16, the increasing $P e$ results in the enhancement of $\omega(\eta)$. Moreover, a maximum value of $\omega(\eta)$ increases with $P e$ and appearance of the maximum value is delayed as $P_{e}$ increases. The influence of $S c$ on $\omega(\eta)$ is opposite to that of $P_{e}$ as plotted in Fig. 17.

Fig. 16. Density of motile microorganisms profiles $\omega(\eta)$ for different values of $P_{e}$ when $\lambda=-2 \operatorname{Pr}=$ $6.2, N_{t}=3.1, N_{b}=3.1, L_{e}=2, S_{c}=1, \varepsilon=-0.5$

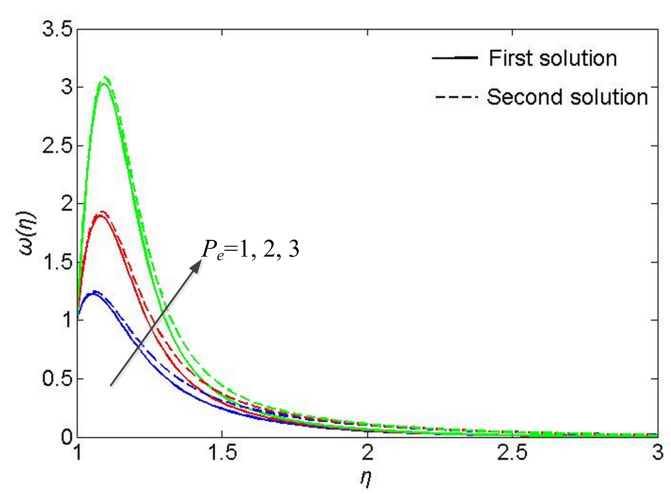

Fig. 17. Density of motile microorganisms profiles $\omega(\eta)$ for different values of $S_{c}$ when $\lambda=-2 \operatorname{Pr}=$ 6.2, $N_{t}=3.1, N_{b}=3.1, L_{e}=2, P_{e}=1, \varepsilon=-0.5$

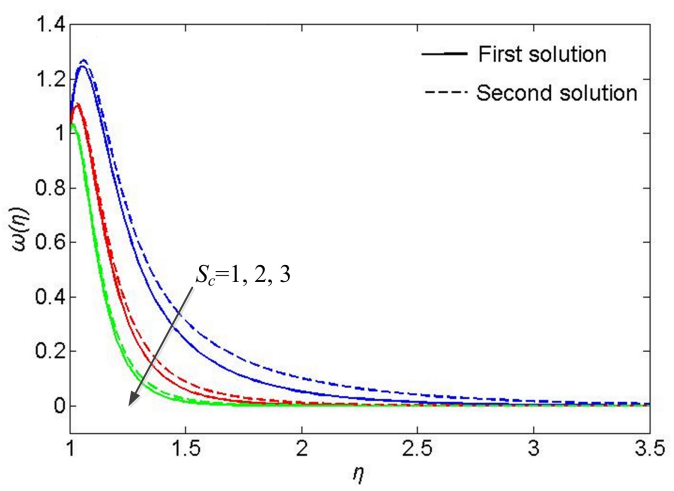




\section{Conclusions}

In the present study, the problem of the unsteady flow over an expanding cylinder in a nanofluid containing gyrotactic microorganisms has been investigated. Different from the previous works, here we first build the unsteady nanofluid model containing gyrotactic microorganisms in cylindrical coordinates. The major conclusions are listed below:

- Dual solutions exist for both stretching $(\varepsilon>0)$ and shrinking cases $\left(\varepsilon_{c}<\varepsilon<0\right)$ with curves which bifurcate at the critical values $\varepsilon_{c}$.

- The range of the dual solutions increases with the strength of the expansion.

- A more rapidly expanding cylinder can generate higher skin friction coefficients and local Nusselt number, but lead to smaller local Sherwood number and local density of the motile microorganisms at the surface.

- Increase in the strengths of Brownian motion effect results in the increase of local Sherwood number and local density of the motile microorganisms, but the opposite effect is reported for the thermophoresis parameter.

- The concentrate of microorganisms is found to increase with higher bioconvection Peclet number and lower Schmidt number.

\section{Acknowledgement}

This work is supported by the National Natural Science Foundation of China (Grant No. 51305080).

\section{References}

1. S.U.S. Choi. ASME Fluid Eng. Division, 231, 99 (1995).

2. S.M. Aminossadati and B. Ghasemi. Eur. J. Mech. B. Fluids, 28, 630 (2009). doi:10.1016/j.euromechflu.2009.05.006.

3. S.K. Das, S.U.S. Choi, W. Yu, and T. Pradeep. Nanofluids: science and technology. John Wiley \& Sons, Hoboken, NJ. 2007.

4. D.A. Nield and A. Bejan. Convection in Porous Media. Springer Science \& Business Media. 2013.

5. J. Buongiorno. J. Heat Transfer, 128, 240 (2006). doi:10.1115/1.2150834.

6. S. Kakaç and A. Pramuanjaroenkij. Int. J. Heat Mass Transfer, 52, 3187 (2009). doi:10.1016/j.ijheatmasstransfer.2009.02.006.

7. K.V. Wong and O. De Leon. Adv. Mech. Engineering, 2010 (2010). doi:10.1155/2010/519659.

8. J. Fan and L.Wang. J. Heat Transfer, 133, 040801 (2011). doi:10.1115/1.4002633.

9. O.Mahian, A. Kianifar, S.A. Kalogirou, I. Pop and S. Wongwises. Int. J. Heat Mass Transfer, 57, 582 (2013). doi:10.1016/j.ijheatmasstransfer.2012.10.037.

10. A.M. Hussein, K.V. Sharma, R.A. Bakar and K. Kadirgama. Renewable Sustainable Energy Rev. 29, 734 (2014). doi:10.1016/j.rser.2013.08.014.

11. R.A. Mandi, H.A. Mohammed, K.M. Munisamy and N.H. Saeid. Renewable Sustainable Energy Rev. 41, 715 (2015). doi:10.1016/-j.rser.2014.08.040.

12. A.V. Kuznetsov. Int. Commun. Heat Mass Transfer, 37, 1421 (2010). doi:10.1016/j.icheatmasstransfer.2010.08.015.

13. L. Tham, R. Nazar and I. Pop. Int. J. Heat Mass Transfer, 62, 647 (2013). doi:10.1016/j.ijheatmasstransfer.2013.03.012.

14. K. Zaimi, A. Ishak and I. Pop. J. Heat Transfer, 136, 41705 (2014). doi:10.1115/1.4026011.

15. W.A. Khan and O.D. Makinde. Z.H. Khan, Int. J. Heat Mass Transfer, 74, 285 (2014). doi:10.1016/j.ijheatmasstransfer.2014.03.026.

16. W.A. Khan and O.D. Makinde. Int. J. Therm. Sci., 81, 118 (2014). doi:10.1016/j.ijthermalsci.2014.03.009.

17. W.N. Mutuku and O.D. Makinde. Comput. Fluids, 95, 88 (2014). doi:10.1016/j.compfluid.2014.02.026.

18. H. Xu and I. Pop. Eur. J. Mech. B. Fluids, 46, 37 (2014). doi:10.1016/j.euromechflu.2014.02.005.

19. R. Nazar, L. Tham, I. Pop and D.B. Ingham. Transp. Porous Media, 86, 517 (2011). doi:10.1007/s11242-010-9637-1.

20. T. Grosan and I. Pop. Int. J. Heat Mass Transfer, 54, 3139 (2011). doi:10.1016/j.ijheatmasstransfer.2011.04.018.

21. L. Tham, R. Nazar and I. Pop. Int. J. Numer. Methods Heat Fluid Flow, 22, 576 (2012). doi:10.1108/09615531211231253.

22. L. Tham, R. Nazar and I. Pop. J. Heat Transfer, 135, 102601 (2013). doi:10.1115/1.4024387.

23. A.M. Rashad, A.J. Chamkha and M. Modather. Comput. Fluids, 86, 380 (2013). doi:10.1016/j.compfluid.2013.07.030.

24. A.M. Rashad, S. Abbasbandy and A.J. Chamkha. J. Heat Transfer, 136, 22503 (2014). doi:10.1115/1.4025559.

25. V.R. Prasad, S.A. Gaffar and O.A. Beg. J. Thermophys Heat Transfer, 29, 127 (2015). doi:10.2514/1.T4396.

26. S.M.M. EL-Kabeir, A.J. Chamkha and A.M. Rashad. J. Porous Media, 17, 269 (2014). doi:10.1615/JPorMedia.v17.i3.70.

28. H.R. Ashorynejad, M. Sheikholeslami, I. Pop and D.D. Ganji. Heat Mass Transfer, 49, 427 (2013). doi:10.1007/s00231-0121087-6.

29. K. Zaimi, A. Ishak and I. Pop. Int. J. Heat Mass Transfer, 68, 509 (2014). doi:10.1016/j.ijheatmasstransfer.2013.09.047.

30. E.M.A. Elbashbeshy, T.G. Emam and M.S. Abdel-wahed. J. Mech. Sci. Technol., 27, 3843 (2013). doi:10.1007/s12206-0130928-7.

31. A.J. Chamkha, A.M. Rashad and A.M. Aly. Meccanica, 48, 71 (2013). doi:10.1007/s11012-012-9584-8.

32. S. Sarkar, S. Ganguly and G. Biswas. Int. J. Heat Mass Transfer, 55, 4783 (2012). doi:10.1016/j.ijheatmasstransfer.2012.04.046.

33. T. Fang, J. Zhang and Y. Zhong. Commun. Nonlinear Sci. Numer. Simul., 17, 3124 (2012). doi:10.1016/j.cnsns.2011.12.013.

34. A.V. Kuznetsov and D.A. Nield. Int. J. Therm. Sci. 49, 243 (2010). doi:10.1016/j.ijthermalsci.2009.07.015.

35. A.V. Kuznetsov and D.A. Nield. Int. J. Therm. Sci. 77, 126 (2014). doi:10.1016/j.ijthermalsci.2013.10.007.

36. W.M.K.A. Wan Zaimi, A. Ishak and I. Pop. Journal of King Saud University-Science, 25, 143 (2013). doi:10.1016/j.jksus.2012.11.005 . 


\section{List of symbol}

$a(t)$

$b$

C

$C_{f}$

$C_{w}$

$C_{\infty}$

$D_{B}$

$D_{n}$

$D_{T}$

f

$j$

$k$

$L_{e}$

$N$

$N_{B}$

$N n_{x}$

$N_{t}$

$N u_{x}$

$N_{w}$

$N_{\infty}$

$p$

$P_{e}$

$P_{r}$

$S_{c}$

$S h_{x}$

$t$

$T$

$T_{w}$

$T_{\infty}$

$v_{r}$

$v_{z}$

$v_{z} w$

V

$W_{c}$

$r, z$

$\alpha$

$\beta$

$\Delta C$

$\varepsilon$

$\eta$

$\theta$

$\lambda$

$\mu$

$\nu$

$\rho$

$(\rho c)_{f}$

$(\rho c)_{p}$

$\tau$

$\phi$

$\omega$
$\nabla^{2}$

Subscripts

\author{
fluid \\ nanoparticle \\ wall condition \\ ambient condition \\ differentiation with respect to $\eta$
}

radius of cylinder

positive constant

chemotaxis constant

nanoparticle concentration

skin friction coefficient

wall nanoparticle concentration

ambient nanoparticle concentration

Brownian diffusion coefficient

diffusivity of microorganisms

thermophoretic diffusion coefficient

dimensionless stream function

flux of microorganisms

thermal conductivity

Lewis number

concentration of microorganisms

Brownian motion parameter

local density number of the motile microorganisms

thermophoresis parameter

local Nusselt number

wall concentration of microorganisms

ambient concentration of microorganisms

pressure

bioconvection Peclet number

Prandtl number

Schmidt number

local Sherwood number

time

temperature inside the boundary layer

wall temperature

ambient temperature

velocity components along the r-axes

velocity components along the z-axes

wall velocity components along the z-axes

velocity vector of nanofluid flow

maximum cell swimming speed

coordinates in the radial and axial directions, respectively

thermal diffusivity

strength of expansion/contraction

characteristic nanoparticle volume fraction

stretching/shrinking parameter

similarity variable

dimensionless temperature

unsteady parameter

dynamic viscosity

kinematic viscosity

fluid density

heat capacity of the fluid

heat capacity of the nanoparticle

ratio between the heat capacity of nanoparticle and fluid

dimensionless nanoparticle concentration

dimensionless concentration of microorganisms

Laplacian operator 